\title{
Consumo alimentar de crianças em municípios de baixo índice de desenvolvimento humano no Nordeste do Brasil ${ }^{1}$
}

\author{
Food consumption of children from cities \\ with a low human development index \\ in the Brazilian Northeast
}

Emilia Chagas COSTA

Silvia Patrícia de Oliveira SILVA²

José Rodolfo Mendonça de LUCENA²

Malaquias BATISTA FILHO²

Pedro Israel Cabral de LIRA ${ }^{2}$

Marisilda de Almeida RIBEIRO²

Mônica Maria OSÓRIO²

RE S U M O

\section{Objetivo}

Este trabalho tem como objetivo analisar o consumo alimentar de crianças em Gameleira, na Zona da Mata Meridional de Pernambuco, e em São João do Tigre, no Semi-Árido da Paraíba, municípios do Nordeste do Brasil.

\section{Métodos}

Trata-se de estudo transversal envolvendo crianças menores de dois anos, das quais 238 em Gameleira, e 207 em São João do Tigre. O consumo alimentar foi registrado utilizando o método recordatório de 24 horas. Os nutrientes foram analisados utilizando as Dietary Referency Intakes.

\section{Resultados}

O consumo médio de energia e da maioria dos nutrientes esteve acima das ingestões de referência, em todas as faixas etárias e em ambos os municípios. A maioria dos valores de prevalência de risco de inadequação dos

\footnotetext{
1 Artigo elaborado a partir da dissertação de E.C. COSTA, intitulada "Consumo alimentar de crianças menores de dois anos de municípios de baixo índice de desenvolvimento humano do Nordeste". Universidade Federal de Pernambuco; 2008. Apoio: Conselho Nacional de Desenvolvimento Científico e Tecnológico (processos n. 502955/03-1 e 502952/03-2), Edital MCT/MESA/CNPq/CT Agronegócio 01/2003.

2 Universidade Federal de Pernambuco, Departamento de Nutrição, Programa de Pós-Graduação em Nutrição. Av. Moraes Rego, s/n., Cidade Universitária, 50670-901, Recife, PE, Brasil. Correspondência para/Correspondence to: M.M. OSÓRIO. E-mail: <mosorio@ufpe.br>
} 
396 | E.C. COSTA et al.

nutrientes foram elevados, principalmente de ferro e zinco, nas crianças entre 7 e 11 meses. O município de São João do Tigre apresentou prevalência de risco, com inadequação em todas as faixas etárias e nutrientes analisados, com exceção da proteína e do ferro na faixa etária entre 12 e 23 meses, embora sem diferença estatisticamente significante.

\section{Conclusão}

A situação nutricional da população estudada ainda preocupa devido às acentuadas prevalências de risco de inadequação dos nutrientes. Portanto, é necessária a elaboração de estratégias que reforcem e promovam a segurança alimentar e nutricional para essas populações, a fim de evitar o risco de instalação de distúrbios nutricionais.

Termos de Indexação: Consumo de alimentos. Criança. Nutrientes.

\section{A B S T R A C T}

\section{Objective}

This study analyzes the food consumption of children from Gameleira, located in the Southern Forest Area of Pernambuco, and from São João do Tigre, located in the semiarid region of Paraíba, in the Brazilian Northeast.

\section{Methods}

This cross-sectional study included 238 children from Gameleira and 207 from São João do Tigre aged <2 years. Food consumption was investigated using a 24-hour dietary recall. Nutrient intake adequacy was classified according to the Dietary Reference Intakes.

\section{Results}

Mean intakes of energy and most nutrients were above the reference values for all ages in both cities. The prevalences of risk of inadequate nutrient intake were high for most nutrients, especially iron and zinc, in children aged 7 to 11 months. São João do Tigre presented higher said prevalences for most studied age groups and nutrients, except for protein and iron in children aged 12 to 23 months, although the difference was not statistically significant.

\section{Conclusion}

The nutrition situation of this population is still of concern because of the high prevalence of risk of inadequate nutrient intake. Therefore, strategies that promote and ensure food and nutrition security are needed for these populations to prevent risk of nutrition disorders.

Indexing terms: Food consumption. Child. Nutrients.

\section{N T R O D U ÇÃ O}

Em nível individual e, principalmente, em escala epidemiológica, a situação de saúde e nutrição da criança reflete, com notável acuidade, as condições de vida da família e da população, expressando o perfil quali-quantitativo de consumo alimentar, a salubridade do micro e macro ambiente, a natureza e o grau dos cuidados dispensados à criança, estreitamente relacionados com os níveis de educação materna e renda da família'. Reunidas numa compreensão mais abrangente de ecossistemas da vida, essas condições são cruciais para modelar o padrão de morbimortalidade e determinar o processo de desenvolvimento da criança, podendo inclusive se prolongar, como efeitos residuais, para todo ciclo vital².

Nessa perspectiva, um aspecto se torna singularmente relevante: o período crítico dos dois primeiros anos de vida, pela elevada vulnerabilidade alimentar da criança, num momento de demandas biológicas intensas e modificações substanciais da alimentação, ou seja, a rápida transição do aleitamento materno para a alimentação habitual da família. Nessa transição, notadamente nas populações de baixas condições socioeconômicas, restrições e impropriedades alimentares conjugadas com o estresse das doenças, sobretudo de natureza infecciosa, estabelecem 
situações de risco que podem se manifestar na ocorrência de processos carenciais, como a desnutrição energético-proteica, as anemias, a hipovitaminose A e outras 3,4 .

Por outro lado, a inadequação alimentar também pode se caracterizar por excesso de energia e nutrientes, ocasionando distúrbios como a obesidade, diabetes e hipertensão arterial, que, além de atingir a população adulta, também começam a surgir em crianças. Esses problemas na infância, juntamente com as condições ambientais e comportamentais, podem ter efeitos importantes na saúde do indivíduo quando adulto 4,5 .

As crianças do Nordeste brasileiro, especialmente nas áreas da Zona da Mata e do Semi-Árido, aqui representadas por dois municípios classificados entre os de mais baixo Índice de Desenvolvimento Humano (IDH) da Região Nordeste, que é a mais pobre do Brasil, podem ser consideradas representativas dos grandes desafios para o desenvolvimento de políticas públicas na área de segurança alimentar e nutricional. Dessa maneira, torna-se pertinente e até imprescindível o conhecimento da dieta das populações que vivem em condições de marcante precariedade socioambiental e assim, expostas ao risco da insegurança alimentar e suas consequências.

Nesse contexto, as avaliações dietéticas do consumo alimentar são valiosas para identificar a participação dos nutrientes na dieta e estimar a sua adequação frente aos valores de referência ${ }^{6,7}$ e, desse modo, permitir o diagnóstico e planejamento de intervenções, fundamentando a promoção de ações globais e específicas para a correção da insegurança alimentar.

Nesse sentido, este estudo tem como objetivo analisar o consumo alimentar de crianças menores de dois anos em Gameleira, Zona da Mata Meridional de Pernambuco, e em São João do Tigre, Semiárido da Paraíba, no Nordeste do Brasil.

\section{M É T O D O S}

Este estudo é parte da pesquisa "Avaliação da situação alimentar e nutricional e seus fatores determinantes em conglomerados urbanos e rurais da Zona da Mata e Semiárido do Nordeste", realizada em Gameleira e São João do Tigre, municípios da Zona da Mata do Estado de Pernambuco e Semiárido da Paraíba, respectivamente. Os dois municípios foram selecionados em função do baixo Índice de Desenvolvimento Humano (IDH de 0,590 e 0,527, respectivamente) ${ }^{8}$ e da perspectiva posterior de implantação de projetos de intervenção, voltados para os objetivos básicos de segurança alimentar e nutricional.

Gameleira pertence à Zona da Mata, notabilizando-se pelas condições de marcante pobreza de sua população, com problemas de nutrição e elevada incidência de doenças infecciosas e parasitárias. Dependente da atividade sucroalcooleira, essa situação de vida já se prolonga por vários séculos, influenciando diretamente nos processos econômicos e sociais do campo e da cidade, com poucas alternativas para contrabalançar o domínio monopolista da cana-de-açúcar e seus ciclos de desemprego em massa. O município de São João do Tigre, por sua vez, pertence ao Semi-Árido do Estado da Paraíba, fazendo parte do maior espaço geográfico contínuo de pobreza do Brasil. Trata-se de uma mesorregião exposta à ocorrência cíclica das grandes estiagens, que resultam em fracassos cruciais da produção agropecuária e no desencadeamento de crises alimentares agudas de grandes proporções, até tempos recentes.

O desenho do estudo aqui relatado foi do tipo transversal, compreendendo populações urbanas e rurais dos dois municípios, representando áreas delimitadas, na perspectiva de funcionar como sítio-sentinela para o acompanhamento posterior da situação alimentar e seus fatores condicionantes. Climaticamente diferenciados e configurados sistemas produtivos também distintos, os municípios de Gameleira e São João do Tigre caracterizam áreas potencialmente críticas com relação à segurança alimentar e nutricional, no contexto do Nordeste do Brasil.

Com idade inferior a dois anos, objeto deste estudo, as amostras totalizaram 238 crianças 
em Gameleira e 207 em São João do Tigre, após a exclusão daquelas que estavam em aleitamento materno exclusivo ou predominante (leite materno mais água ou chá) e dos inquéritos de consumo que apresentavam informações inconsistentes (30 casos em Gameleira e 24 em São João do Tigre).

O inquérito de consumo alimentar foi realizado por nutricionistas e estudantes de nutrição, utilizando o método recordatório de 24 horas. 0 formulário foi aplicado junto à mãe biológica ou adotiva, ou a outra pessoa adulta responsável pelo cuidado da criança, tendo como referência o que comeu "desde a meia-noite do dia de anteontem até a meia-noite de ontem". A entrevista incluiu questões sobre todos os horários e refeições (desjejum, lanches, almoço, jantar e ceia), alimentos consumidos, formas de preparo e marcas comerciais (alimentos industrializados), compreendendo quantidades preparadas, oferecidas e consumidas pela criança, em medidas caseiras e medidas de volume. A quantidade dos alimentos foi informada segundo o tamanho, para frutas e vegetais (pequeno, médio e grande); tamanho do prato (pequeno, médio e grande; fundo ou raso), tamanho da colher (pequena, média e grande) e quantidade de alimento (rasa, normal, cheia), para alimentos preparados, leite em pó, açúcar e massas; tamanho do copo ou xícara e a quantidade de alimentos líquidos neles contida. Para maior precisão da quantidade consumida, foram pesadas in loco e registradas as quantidades de determinados alimentos iguais às utilizadas nas preparações do dia anterior. Para mensuração da quantidade, foi utilizada uma balança eletrônica, com capacidade de $5 \mathrm{~kg}$ e graduações de $1 \mathrm{~g}$. Os líquidos foram mensurados em proveta de $500 \mathrm{~mL}$.

Tendo em vista que as populações dos dois municípios eram semelhantes em termos de condições socioeconômicas e demográficas, mais duas repetições do inquérito recordatório de 24 horas foram realizadas numa subamostra de 19\% das crianças do município de Gameleira, com a finalidade de determinar a variação intrapessoal do consumo alimentar da população. Os dois últimos recordatórios foram realizados em dias não consecutivos, repetindo o procedimento adotado no primeiro dia.

As medidas caseiras das porções relatadas nos questionários de consumo alimentar das crianças foram calculadas por nutricionistas e alunos de iniciação científica do Curso de Nutrição da Universidade Federal de Pernambuco.

Posteriormente, os valores per capita foram digitados utilizando o software Virtual Nutri. Para análise da composição química dos alimentos regionais inexistentes no software, foram usadas como referência a Tabela de Composição de Alimentos do Instituto Brasileiro de Geografia e Estatística (IBGE) ${ }^{9}$ e a Tabela Brasileira de Composição de Alimentos $(\mathrm{TACO})^{10}$. O consumo do leite materno foi determinado pela frequência das mamadas, considerando-se o volume de cada uma delas de acordo com a idade da criança, informação fornecida pelo software Virtual Nutri.

O processamento dos dados e a análise estatística foram realizados no programa Statistical Package for Social Science, versão 12.0 (SPSS Inc, Chicago, IL, USA). Os nutrientes estudados foram: carboidrato, proteína, gordura, ferro, cálcio, zinco, vitamina $A$ e $C$. Para estimar a ingestão habitual das populações de crianças menores de dois anos nos dois municípios, os valores da ingestão de calorias, macro e micronutrientes foram ajustados pela equação proposta pelo US National Academy of Science, Subcomitte on Criteria for Dietary Evaluation ${ }^{11}$. Esse ajuste foi realizado por meio das informações coletadas em mais dois inquéritos recordatórios de $24 \mathrm{~h}$, em dias da semana não consecutivos, em uma subamostra de 45 crianças do município de Gameleira. A normalidade da distribuição das variáveis foi testada mediante a aplicação do teste de Kolmogorov Sminorf, e aqueles nutrientes que não apresentaram distribuição normal foram convertidos para o seu logaritmo natural, sendo a sua normalidade testada novamente. Foi necessário utilizar essa transformação no grupo dos macronutrientes, apenas para a 
gordura nas faixas etárias de 0 a 6 meses e acima de um ano, no município de São João do Tigre.

Com relação aos micronutrientes zinco e ferro, na faixa etária de 0 a 6 meses, e a vitamina A, nas crianças de 12 a 23 meses, em São João do Tigre, mesmo após a sua transformação em logaritmo natural, os resultados ainda apresentaram distribuição assimétrica, o que impossibilitou o cálculo da sua prevalência de inadequação. Para todos os micronutrientes, optou-se por trabalhar com os valores medianos e seus percentis 25 e 75. Os dados relativos aos macronutrientes foram descritos em médias ajustadas e seus respectivos desvios-padrão.

A prevalência de inadequação, referente ao consumo de macro e micronutrientes, foi considerada em relação aos valores das Dietary Reference Intakes (DRI), propostos pelo Food and Nutrition Board/Institute of Medicine, sendo a população dividida em três faixas de idade ( 0 a 6 meses, 7 a 11 meses e 12 a 23 meses). A prevalência de inadequação da ingestão de energia corresponde à proporção de indivíduos cujo consumo estava abaixo da Estimated Energy Requirement (EER) calculada para a faixa etária. A prevalência de inadequação de proteínas, carboidratos, zinco, ferro, vitamina $A$ e vitamina $C$ corresponde à proporção de indivíduos abaixo da Estimated Average Requirement (EAR). Os cálculos de EER foram realizados a partir das equações para predição de gasto energético total nos diferentes estágios de vida, considerando idade, sexo, peso e estatura. Para os nutrientes que ainda não possuem os valores de referência da EAR, a comparação da referência foi realizada com base na Ingestão Adequada (AI). Para a gordura, na faixa etária de 12 a 23 meses não há valor de referência ${ }^{12-15}$.

O teste $t$-Student foi aplicado para analisar possíveis diferenças estatísticas entre as duas populações, comparando-se as médias do consumo de energia e macronutrientes, enquanto na comparação do consumo mediano entre os micronutrientes foi utilizado o teste de Mann-Whitney. Para verificar diferenças entre as prevalências de inadequação de consumo dos nutrientes entre os dois municípios, foi utilizado o teste qui-quadrado.

A pesquisa "Avaliação da Situação Alimentar e Nutricional e seus Fatores Determinantes em Conglomerados Urbanos e Rurais do Nordeste" foi aprovada pelo Comitê de Ética e Pesquisa em Seres Humanos do Instituto Materno Infantil de Pernambuco Prof. Fernando Figueira, atendendo às normas da Resolução 196/96, do Conselho Nacional de Saúde.

\section{RES U LT A D O S}

O presente estudo investigou 445 crianças, das quais 238 residentes no município de Gameleira e 207, em São João do Tigre. Em Gameleira, $51,7 \%$ das crianças eram do sexo masculino, $59,8 \%$ das famílias apresentaram renda mensal menor do que $1 / 4$ do salário-mínimo, e 34,0\% das crianças estavam em aleitamento materno. Em São João do Tigre, $41,1 \%$ das crianças eram do sexo masculino, 55,1\% das famílias apresentaram renda mensal menor do que $1 / 4$ do salário-mínimo, e $39,1 \%$ das crianças estavam em aleitamento materno.

Ao comparar os valores médios de energia e macronutrientes consumidos pelas crianças menores de dois anos nos municípios de Gameleira e São João do Tigre com os valores de referência das Dietary Reference Intakes (DRI), observa-se que todos os nutrientes apresentaram valores médios acima da recomendação e que praticamente não existia diferença no consumo dos macronutrientes entre os dois municípios, com exceção da proteína na faixa entre 0 e 6 meses, e da gordura na faixa entre 12 e 23 meses, sendo o primeiro nutriente mais consumido em Gameleira e o segundo, em São João do Tigre (Tabela 1).

A maioria dos micronutrientes apresentou valor de consumo acima das referências (Tabela 2). Entretanto, com relação ao consumo de vitamina $A$, as crianças entre 7 e 11 meses, nos dois municípios, apresentaram valores medianos abaixo da ingestão adequada (AI). O mesmo 
$400 \mid$ E.C. COSTA et al.

Tabela 1. Análise comparativa das médias de energia e macronutrientes consumidos por crianças menores de dois anos, nos municípios de Gameleira (PE) e São João do Tigre (PB), 2005.

\begin{tabular}{|c|c|c|c|c|c|}
\hline \multirow{2}{*}{ Especificações } & \multicolumn{2}{|c|}{ Gameleira (PE) } & \multicolumn{2}{|c|}{ São João do Tigre (PB) } & \multirow{2}{*}{ Valor DR } \\
\hline & Média & DP & Média & DP & \\
\hline \multicolumn{6}{|c|}{0 - 6 meses } \\
\hline & \multicolumn{2}{|c|}{$(n=49)$} & \multicolumn{2}{|c|}{$(n=68)$} & \\
\hline Energia (kcal) & 832,9 & 345,7 & 715,4 & 329,8 & $612^{1}$ \\
\hline Carboidratos (g) & 114,1 & 63,0 & 86,5 & 50,7 & $60^{3}$ \\
\hline Proteínas (g) & $20,9^{a}$ & 9,6 & $19,4^{\mathrm{a}}$ & 13,2 & $9,1^{3}$ \\
\hline Gordura (g) & 31,9 & 13,3 & 33,0 & 14,5 & $31^{3}$ \\
\hline \multicolumn{6}{|c|}{ 7-11 meses } \\
\hline & \multicolumn{2}{|c|}{$(n=62)$} & \multicolumn{2}{|c|}{$(n=32)$} & \\
\hline Energia (kcal) & 1089,6 & 538,9 & 985,2 & 498,7 & $721^{1}$ \\
\hline Carboidratos (g) & 162,4 & 90,4 & 139,9 & 80,2 & $95^{3}$ \\
\hline Proteínas (g) & 30,4 & 15,7 & 28,7 & 16,9 & $10^{2}$ \\
\hline Gordura (g) & 34,1 & 13,0 & 34,9 & 16,1 & $30^{3}$ \\
\hline \multicolumn{6}{|c|}{12 - 23 meses } \\
\hline & \multicolumn{2}{|c|}{$(n=127)$} & \multicolumn{2}{|c|}{$(n=107)$} & \\
\hline Energia (kcal) & 1278,4 & 595,8 & 1190,0 & 467,6 & $1051^{1}$ \\
\hline Carboidratos (g) & 194,0 & 91,0 & 160,5 & 61,2 & $100^{2}$ \\
\hline Proteínas (g) & 39,8 & 21,7 & 38,6 & 17,2 & $11^{2}$ \\
\hline Gordura (g) & $38,3^{\mathbf{b}}$ & 18,9 & $44,9^{\mathbf{b}}$ & 21,8 & - \\
\hline
\end{tabular}

Teste $t$ de Student: a: $p \leq 0,05,{ }^{b}$ : $p \leq 0,01 ;{ }^{1}$ Valores de DRI determinado por EER; ${ }^{2}$ Valores de DRI determinado por EAR; ${ }^{3}$ Valores de DRI determinado por Al.

DP: desvio-padrão; DRI: dietary reference intakes; EER: estimated energy requirement; EAR: estimated average requirement; Al: ingestão adequada.

Tabela 2. Análise comparativa do consumo mediano de micronutrientes em crianças menores de dois anos, nos municípios de Gameleira (PE) e São João do Tigre (PB), 2005.

\begin{tabular}{|c|c|c|c|c|c|}
\hline \multirow{2}{*}{ Especificações } & \multicolumn{2}{|c|}{ Gameleira (PE) } & \multicolumn{2}{|c|}{ São João do Tigre (PB) } & \multirow{2}{*}{ Valor DRI } \\
\hline & Mediana & $(25 \%-75 \%)$ & Mediana & $(25 \%-75 \%)$ & \\
\hline \multicolumn{6}{|c|}{$0-6$ meses } \\
\hline & \multicolumn{2}{|c|}{$(n=49)$} & \multicolumn{2}{|c|}{$(n=68)$} & \\
\hline Vitamina A (mcg) & $494,7^{b}$ & $(342,9-723,7)$ & $382,9^{\mathbf{b}}$ & $(226,3-480,6)$ & $400^{2}$ \\
\hline Vitamina C (mcg) & 30,9 & $(20,2-48,6)$ & 30,8 & $(21,0-38,6)$ & $40^{2}$ \\
\hline Ferro $(\mathrm{mg})$ & 3,0 & $(0,87-6,5)$ & 2,3 & $(0,2-5,7)$ & $0,27^{2}$ \\
\hline Zinco (mg) & 2,8 & $(1,6-3,6)$ & 2,0 & $(1,1-3,8)$ & $2,0^{2}$ \\
\hline Cálcio (mg) & 640,9 & $(399,7-1006,0)$ & 457,5 & $(222,3-1134,6)$ & $210^{2}$ \\
\hline \multicolumn{6}{|c|}{$7-11$ meses } \\
\hline & \multicolumn{2}{|c|}{$(n=62)$} & \multicolumn{2}{|c|}{$(n=32)$} & \\
\hline Vitamina A (mcg) & $461,1^{b}$ & $(303,8-791,5)$ & $293,8^{\mathbf{b}}$ & $(192,5-373,7)$ & $500^{2}$ \\
\hline Vitamina C (mcg) & 33,3 & $(19,5-76,1)$ & 36,8 & $(22,4-63,1)$ & $50^{2}$ \\
\hline Ferro $(\mathrm{mg})$ & 5,3 & $(1,9-11,5)$ & 4,0 & $(1,3-10,0)$ & $6,9^{1}$ \\
\hline Zinco (mg) & 3,0 & $(1,5-4,9)$ & 2,8 & $(1,3-3,7)$ & $2,5^{1}$ \\
\hline \multirow[t]{3}{*}{ Cálcio (mg) } & 854,8 & $(406,6-1330,1)$ & 843,0 & $(275,5-1326,0)$ & $270^{2}$ \\
\hline & \multicolumn{4}{|c|}{$12-23$ meses } & \\
\hline & \multicolumn{2}{|c|}{$(n=127)$} & \multicolumn{2}{|c|}{$(n=107)$} & \\
\hline Vitamina A (mcg) & $476,5^{b}$ & $(280,0-773,0)$ & $260,2^{\mathbf{b}}$ & $(188,0-363,7)$ & $210^{1}$ \\
\hline Vitamina C (mcg) & 30,1 & $(16,7-76,6)$ & 33,3 & $(22,2-64,9)$ & $13^{1}$ \\
\hline Ferro $(\mathrm{mg})$ & 6,1 & $(2,2-11,7)$ & 6,7 & $(3,6-8,9)$ & $3,0^{1}$ \\
\hline Zinco (mg) & 4,1 & $(2,2-6,0)$ & 3,9 & $(1,95-5,31)$ & $2,5^{1}$ \\
\hline Cálcio (mg) & $921,8^{a}$ & $(511,9-1431,4)$ & $1223,5^{a}$ & $(668,3-1914,0)$ & $500^{2}$ \\
\hline
\end{tabular}

Teste de Mann-Whitney: ${ }^{\text {a: }} p \leq 0,05,{ }^{\text {b}}: p \leq 0,001 ;{ }^{1}$ Valores de DRI determinado por EAR; ${ }^{2}$ Valores de DRI determinado por Al. DRI: dietary reference intakes; EAR: estimated average requirement; Al: ingestão adequada. 
comportamento foi verificado nas crianças menores de 7 meses no município de São João do Tigre. No que diz respeito à vitamina $C$, seus valores medianos de consumo estiveram acima da $\mathrm{Al}$ apenas nas crianças de 12 a 23 meses, nos dois municípios.

Em relação ao ferro, as crianças de 7 a 11 meses nos dois municípios apresentaram o consumo mediano abaixo da EAR, enquanto o consumo mediano de zinco e de cálcio esteve acima das referências em todas as faixas etárias, nos dois municípios.

Ao comparar os dois municípios, o consumo mediano de vitamina A e de zinco foi maior em Gameleira, em todas as faixas etárias. Já o consumo mediano de cálcio foi maior em São João do Tigre para as crianças maiores de 1 ano. Tais resultados apresentaram diferenças estatísticas significativas, com exceção dos valores para o zinco.

A prevalência de inadequação de energia nas crianças menores de 7 meses foi maior no município de São João do Tigre, representando $32,6 \%$ de ocorrência, enquanto em Gameleira esse percentual foi de $26,8 \%$, embora sem diferença estatisticamente significativa (resultados não apresentados).

Para as crianças de 7 a 11 meses, o mesmo comportamento foi observado com as prevalências de inadequação de energia, proteína, zinco e ferro, maiores no município de São João do Tigre quando comparadas às do município de Gameleira, embora essas diferenças não sejam estatisticamente significativas (Figura 1).

Na faixa etária de 12 a 23 meses, os valores de inadequação de energia foram praticamente iguais nos dois municípios. As prevalências de inadequação de proteína e ferro foram maiores no município de Gameleira, enquanto as de carboidrato, vitamina $C$ e zinco foram maiores em São João do Tigre. Na análise estatística, não houve diferença significativa na prevalência de inadequação do consumo de nutrientes nessa faixa etária entre os dois municípios (Figura 2).

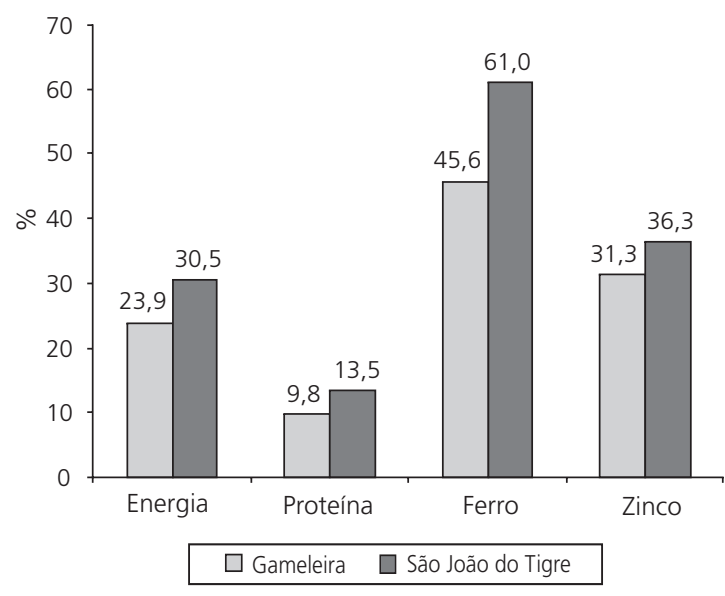

Figura 1. Percentual de inadequação de energia e nutrientes em crianças de 7 a 11 meses, nos municípios de Gameleira (PE) e São João do Tigre (PB), 2005

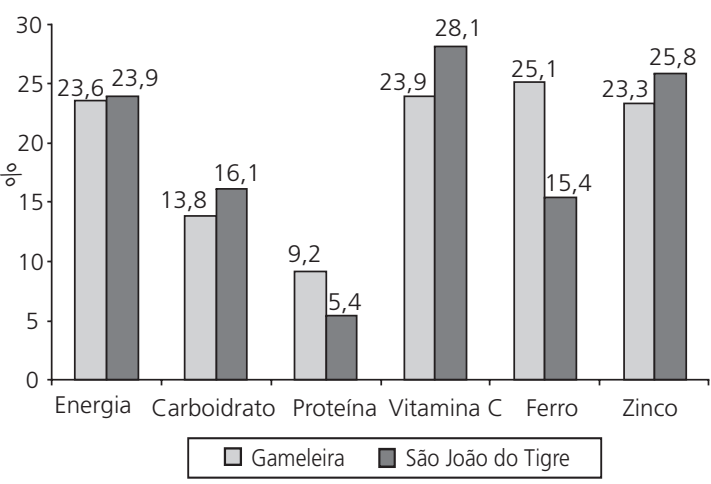

Figura 2. Percentual de inadequação de energia e nutrientes em crianças de 12 a 23 meses, nos municípios de Gameleira (PE) e São João do Tigre (PB), 2005

\section{DIS C U S S Ã O}

As crianças, em especial as menores de dois anos, constituem um grupo de peculiar vulnerabilidade, do ponto de vista de deficiência de macro e micronutrientes, por sua exposição a uma alimentação inadequada, principalmente pelo desmame precoce, introdução errônea da alimentação complementar e necessidades biológicas aumentadas. Essas relações de risco encontram-se mediadas pelas condições de vida: à medida que estas se tornam mais precárias, maiores são as chances para o desenvolvimento da maioria dos agravos nutricionais ${ }^{1,2}$. 
402 | E.C. COSTA et al.

Os resultados do presente estudo demonstram prevalência de inadequação de energia, variando de $23,6 \%$ a 30,5\% entre as crianças de 7 a 23 meses, nos dois municípios estudados. Entretanto, com relação ao carboidrato, salienta-se que a necessidade desse nutriente a partir de um ano é baseada na quantidade mínima de glicose que é utilizada pelo cérebro, sem fonte alternativa de energia, a fim de prevenir uma possível cetose. Portanto, o consumo médio de carboidrato dessas crianças apresenta-se acima dos valores das necessidades mínimas de carboidrato.

De modo geral, verifica-se uma pequena melhora no consumo médio e na prevalência de inadequação de energia nas crianças do Estado de Pernambuco, em comparação com os resultados obtidos em 1997, quando esta última variava entre $28,6 \%$ e $39,6 \%$ nas áreas urbanas, e entre $26,5 \%$ e $55,4 \%$ nas áreas rurais ${ }^{16}$.

Por outro lado, os resultados indicam um consumo médio de macronutrientes acima dos valores de referência das DRI para todas as faixas etárias, nos dois municípios. Isso, associado às prevalências de inadequação relativamente baixas, quando comparadas aos micronutrientes, sugere um padrão alimentar acima da média para a energia, e ao mesmo tempo insuficiente em alguns elementos essenciais, característico de uma dieta monótona, com elevado consumo de açúcar e gordura ${ }^{17}$. Esse fato pode ser evidenciado nos dois municípios, uma vez que o consumo médio de proteína foi superior aos valores das DRI, ressaltando-se que, em relação a todos os nutrientes analisados quanto à prevalência de inadequação, a proteína foi o que apresentou menores valores de risco, corroborando com alguns estudos dietéticos, que demonstram o consumo proteico adequado e/ou com menores prevalências de inadequação, apesar de deficiências de macro e micronutrientes encontradas na alimentação das crianças $^{16,18}$.

Salienta-se que a maioria das crianças aqui estudada estava recebendo alimentação predominantemente à base de leite de vaca. É possível que crianças sem aleitamento exclusivo devam consumir mais proteína por quilo de peso, quando comparadas com crianças em aleitamento mater$\mathrm{no}^{18}$. Esse excesso de ingestão proteica pode resultar em uma série de implicações, incluindo o acúmulo de tecido adiposo subcutâneo, o que leva ao risco de desenvolvimento de doenças crônicas não transmissíveis, na vida adulta ${ }^{19}$.

Efeitos da globalização e hábitos de vida podem estar associados ao consumo de gordura igual ou superior às DRI. Essa alternativa estaria relacionada às campanhas publicitárias que influenciam a escolha dos alimentos, especialmente em famílias de baixa renda, favorecendo a utilização de produtos industrializados oferecidos em larga escala e a baixo custo, geralmente ricos em açúcares simples e gorduras ${ }^{20}$.

Desse modo, as crianças tendem a acompanhar a modificação do cenário de saúde e nutrição da população brasileira, que vem ocorrendo nas últimas três décadas, caracterizada por aumento no consumo de proteínas e gorduras (principalmente as saturadas), excesso de açúcar e consumo insuficiente de frutas e hortaliças, em nível populacional 5 .

Em relação aos micronutrientes, o consumo mediano de ferro em crianças de 7 a 11 meses não atinge a recomendação proposta. Nessa idade, a criança torna-se extremamente vulnerável por ser o período de desmame, associado à introdução de leite de vaca, característico de uma dieta monótona que favorece o aparecimento da anemia ferropriva ${ }^{21}$. As demais faixas etárias demonstraram valores de consumo mediano de ferro superiores às DRI, o que na idade de 0 a 6 meses poderia estar relacionado à utilização do leite materno e à introdução precoce de outros alimentos. Na idade de 12 a 23 meses, a dieta pode estar mais diversificada e com alimentos ricos em ferro; entretanto, essa quantidade de ferro não pode refletir diretamente no estado nutricional da criança, pois nem todo ferro dietético é devidamente absorvido pelo organismo.

No município de Gameleira, 46,3\% das crianças apresentaram anemia, enquanto em São João do Tigre a prevalência foi de $37,1 \%{ }^{22,23}$. Essas 
prevalências chamam atenção, uma vez que, segundo a Organização Mundial da Saúde ${ }^{24}$, prevalências de anemia maiores que 40,0\% têm como causa mais provável a deficiência de ferro dietético. Tomando como base os dados do consumo de ferro deste estudo e a proporção das crianças que estavam abaixo da EAR $(25,1 \%$, em Gameleira, e 15,4\%, em São João do Tigre), é possível supor uma associação dessa inadequação com as altas prevalências de anemia nos dois municípios.

O consumo de vitamina A, com medianas abaixo da recomendação de Ingestão Adequada (Al), principalmente em São João do Tigre, ressalta o fato do Nordeste, principalmente o Semiárido, ser reconhecido como uma extensa área de elevado risco de deficiência de vitamina $A^{25}$. Por outro lado, apesar da grande pobreza, a Zona da Mata, em determinadas estações do ano, seria favorecida por uma maior oferta de vegetais ricos em caroteno, graças ao clima tropical quente e úmido, o que pode estar associado ao consumo dessa vitamina abaixo das recomendações em apenas uma faixa etária.

Todavia, parece que o acesso a frutas e verduras é escasso nos dois municípios, contribuindo para os valores de vitamina $\mathrm{C}$ abaixo das DRI. Esse fato pode ser mais um fator determinante na ocorrência de anemia nessas populações, uma vez que a presença da vitamina $C$, principalmente durante as grandes refeições, facilita a absorção do ferro não-heme pelo organismo.

De acordo com Salles-Costa ${ }^{26}$, o programa utilizado na avaliação dietética do presente estudo subestimaria o zinco, pois o software apresenta um número limitado de alimentos avaliados com a composição desse nutriente, ao lado de diferenças na composição de alguns alimentos. No entanto, a mediana no consumo desse nutriente apresentou-se igual ou acima da recomendação em todas as faixas etárias para os dois municípios, embora fossem verificados percentuais acima dos $20 \%$ da prevalência de inadequação de zinco. Cabe ressaltar que a presença desse nutriente em níveis adequados não assegura sua utilização pelo organismo, pois o consumo de zinco é influenciado por algumas proteínas, como a caseína, e pelo conteúdo de fitatos presentes na dieta, reduzindo a sua biodisponibilidade ${ }^{27}$.

O consumo mediano de cálcio acima das recomendações das DRI provavelmente se deve à introdução precoce do leite de vaca e cabra na alimentação das crianças e ao seu alto consumo. O leite de cabra é especialmente consumido no município de São João do Tigre, por ser produto beneficiado pela cooperativa local e distribuído à população por meio de programa municipal em parceria pelo Governo Federal. Entretanto, o alto consumo de leite de vaca ou de cabra, aliado à pouca adesão de outros alimentos complementares, pode representar risco à população infantil, uma vez que esses alimentos, ricos em proteínas e minerais, em especial o cálcio, interferem na absorção do ferro proveniente do leite materno e de outros alimentos, além de possibilitar ocorrência de micro-hemorragias intestinais ${ }^{21,28}$. Desse modo, as duas populações também podem ter suas prevalências de anemia agravadas por conta desse padrão alimentar.

Apesar dos avanços no campo dos estudos dietéticos, a estimativa do consumo alimentar e nutricional na infância é um desafio, diante da maior variabilidade de erros na mensuração da dieta da criança, em função da dependência dos pais no relato dos alimentos consumidos por elas $^{26}$. Para minimizar as fontes de variabilidade de erro neste estudo, foi realizada a repetição do inquérito em uma subamostra e feita a pesagem in loco das quantidades consumidas de determinados alimentos, iguais às utilizadas nas preparações do dia anterior à entrevista e que estavam disponíveis na residência da criança. Na análise dos dados, foi aplicado o método de cálculo de prevalência de inadequação, a partir da metodologia da "EAR como ponto de corte", na qual o consumo alimentar é ajustado pela variância inter e intrapessoal ${ }^{7}$.

Embora a maioria das crianças tenha apresentado os valores de nutrientes consumidos acima dos valores de referência das DRI, a situação 
404 E.C. costA et al.

alimentar dessa população ainda é preocupante, devido às acentuadas prevalências de inadequação encontradas para os nutrientes analisados.

Diante desse diagnóstico, das condições de pobreza e do contexto histórico que prevalecem nesses dois municípios, essas regiões devem ser prioridades da Política Nacional de Segurança Alimentar e Nutricional. Assim faz-se necessária a elaboração de estratégias que reforcem a área de vigilância alimentar e nutricional para essas crianças, estimulando o aleitamento materno exclusivo e prolongado, bem como ações educativas sustentáveis, incluindo políticas e programas que possam resgatar as distorções já tradicionais de um modelo econômico socialmente excludente.

\section{COLABORADORES}

M.M. OSÓRIO, M. BATISTA FILHO; P.I.C. LIRA e M.A. RIBEIRO participaram da elaboração dos projetos de estudo, instrumentos de coleta de dados, supervisão dos trabalhos de campo, levantamento bibliográfico, análise estatística e redação do trabalho. E.C. COSTA e S.P.O. SILVA participaram da elaboração dos instrumentos de campo, coleta e tabulação dos dados, levantamento bibliográfico, análise estatística e redação do artigo. J.R.M. LUCENA participou da tabulação dos dados e análise estatística.

\section{REFERÊ NCIAS}

1. Assis AMO, Barreto ML, Santos NS, Oliveira LPM, Santos SMC, Pinheiro SMC. Desigualdade, pobreza e condições de saúde e nutrição na infância no Nordeste brasileiro. Cad Saúde Pública. 2007; 23(10):2337-50.

2. Fundo das Nações Unidas para a Infância. Situação da infância brasileira 2006. Brasília: Unicef; 2005.

3. Conde WL, Gigante DP. Epidemiologia da desnutrição infantil. In: Kac G, Sichieri R, Gigante DP, organizadores. Epidemiologia nutricional. Rio de Janeiro: Atheneu; 2007. p.281-95.

4. World Heath Organization. Global strategy on diet, physical activity and health. Fifty Seventh World Health Assembly. Geneva: WHO; 2004. [WHA 57.17].
5. Levy-Costa RB, Sichieri R, Pontes NS, Monteiro CA. Disponibilidade domiciliar de alimentos no Brasil: distribuição e evolução (1974-2003). Rev Saúde Pública. 2005; 39(4):530-40.

6. Barreto SM, Pinheiro ARO, Sichieri R, Monteiro CA, Batista Filho M, Schimdt MI, et al. Análise da estratégia global para alimentação, atividade física e saúde, da Organização Mundial da Saúde. Epidemiol Serv Saúde. 2005; 14(1):41-68.

7. Slater B, Lobo MD, Fisberg RM. Estimating prevalence of inadequate nutrient intake. Rev Saúde Pública. 2004; 38(4):599-605.

8. Programa das Nações Unidas para o Desenvolvimento. Índice de desenvolvimento humano 2000. [acesso 2007 mar 5]. Disponível em: <http://www. pnud.org.br/atlas/tabelas/index.php>.

9. Instituto Brasileiro de Geografia e Estatística. Estudo Nacional da Despesa Familiar - ENDEF. Tabela de composição de alimentos. $4^{a}$ ed. Rio de Janeiro: IBGE; 1996.

10. Universidade Estadual de Campinas. Tabela brasileira de composição de alimentos. Versão 2. Campinas: Unicamp; 2006.

11. Guenther PM, Kott OS, Carriquiry AL. Development of an approach for estimating usual nutrient intake distributions at the population level. J Nutr. 1997; 127(6):1106-12.

12. Institute of Medicine. National Research Council. Dietary reference intakes for energy, carbohydrate, fiber, fat, fatty acids, cholesterol, protein, and amino acids (Macronutrients). Washington (DC): National Academy Press; 2002.

13. Institute of Medicine. National Research Council. Dietary reference intakes for vitamin A, vitamin $\mathrm{K}$, arsenic, boron, chromium, copper, iodine, iron, manganese, molybdenum, nickel, silicon, vanadium, and zinc. Washington (DC): National Academy Press; 2002.

14. Institute of Medicine. National Research Council. Dietary reference intakes for vitamin C, vitamin E, selenium and carotenoids. Washington (DC): National Academy Press; 2002.

15. Institute of Medicine. National Research Council. Dietary reference intakes for calcium, phosphorus, magnesium, vitamin D and fluorido. Washington (DC): National Academy Press; 1999.

16. Fidelis CMF, Osório MM. Consumo alimentar de macro e micronutrientes de crianças menores de cinco anos no Estado de Pernambuco, Brasil. Rev Bras Saúde Mater Infant. 2006; 7(1):61-72.

17. Farias Junior G, Osório MM. Padrão alimentar de crianças menores de cinco anos. Rev Nutr. 2005; 18(6):793-802. doi: 10.1590/S1415-5273200500 0600010. 
18. Menezes, RCE, Osório MM. Consumo energéticoprotéico e estado nutricional de crianças menores de cinco anos, no estado de Pernambuco, Brasil. Rev Nutr. 2007; 20(4):337-47. doi: 10.1590/\$14 15-52732007000400001.

19. Alexy U, Kersting M, Sichet-Hellert W, Manz F, Schoch G. Macronutrient of 3 to 36-minth-old German infants and children: results of the DONALD Study. Dortmund Nutritional and Antropometric Longitudinally Designed Study. Ann Nutr Metab. 1999; 43(1):14-22.

20. Caballero B. Subnutrição e obesidade em países em desenvolvimento. In: Cadernos de estudos desenvolvimento social em debate, n.2. Brasília: Ministério do Desenvolvimento Social e Combate à Fome; 2005. p.10-13.

21. Oliveira MA, Osório MM. Consumo de leite de vaca e anemia ferropriva na infância. J Pediatr (Rio de Janeiro). 2005; 81(5):361-7.

22. Batista Filho M. Avaliação da situação alimentar e nutricional e seus fatores determinantes em conglomerados urbanos e rurais do semi-árido do estado da Paraíba. Brasília: MCT; 2007.

23. Lira PIC. Avaliação da situação alimentar e nutricional e seus fatores determinantes em conglo- merados urbanos e rurais da zona da mata do estado de Pernambuco. Brasília: MCT; 2007.

24. World Health Organization. Iron deficiency anemia: assessment, prevention and control. Geneva: WHO; 2001.

25. Santos LMP, organizador. Bibliografia sobre deficiência de micronutrientes no Brasil 1990-2000: Vitamina A. Brasília: Organização Pan-Americana de Saúde; 2002. v.1.

26. Salles-Costa R, Antunes MM, Mello MA, Sichieri R. Comparação de dois programas computacionais utilizados na estimativa do consumo alimentar em crianças. Rev Bras Epidemiol. 2007; 10(2):267-75.

27. Mafra D, Cozzolino SMF. Importância do zinco na nutrição humana. Rev Nutr. 2004; 17(1):79-87. doi: 10.1590/S1415-52732004000100009.

28. Levy-Costa RB, Monteiro CA. Consumo de leite de vaca e anemia na infância no Município de São Paulo. Rev Saúde Pública. 2004; 38(6):797-803.

Recebido em: 25/11/2009

Versão final reapresentada em: 13/10/2010

Aprovado em: 9/11/2010 
